\title{
CÁC HỢP CHẤT PHYTOSTEROL, TRITERPEN, VÀ ALCOL MẠCH DÀI PHÂN LẬP TỬ LÁ TRÀ ĐÀ LẠT (C amellia dalatensis Luong, Tran \& Hakoda)
}

\section{Nguyễn Thị Tố Uyên ${ }^{\mathrm{a}}$, Trần Thị Thanh Phúc ${ }^{\mathrm{a}}$, Lương Văn Dũng ${ }^{\mathrm{b}}$, Trịnh Thị Điệp $\mathbf{a}^{\mathrm{a}^{*}}$}

\author{
${ }^{a}$ Khoa Hóa hoc, Truờng Đại hoc Đà Lạt, Lâm Đồng, Việt Nam \\ ${ }^{b}$ Khoa Sinh học, Truờng Đại học Đà Lạt, Lâm Đồng, Việt Nam \\ "Tác giả liên hệ: Email: dieptt@dlu.edu.vn
}

Lịch sử bài báo

Nhận ngày 26 tháng 11 năm 2018

Chỉnh sửa ngày 11 tháng 12 năm 2018 | Chấp nhận đăng ngày 14 tháng 12 năm 2018

\section{Tóm tắt}

Tù̀ dịch chiết n-hexan và dichloromethan của lá Trà Mi Đà Lạt (Camellia dalatensis Luong, Tran, \& Hakoda), bằng các phuoong pháp sắc ký kết hợp với các phuoong pháp phổ hiện đại (IR, 1H-NMR, 13C-NMR, DEPT, HSQC, HMBC, và ESI-MS) đã phân lập và nhận dạng được cấu trúc năm hơp chất là spinasterol, stigmasterol, acid oleanolic, docosan, và 1-tricosanol. Đây là lần đầu tiên các hợp chất này được phân lập tù̀ loài C. dalatensis.

Từ khóa: 1-tricosanol; Camellia dalatensis; Oleanolic acid; Spinasterol; Stigmasterol.

DOI: http://dx.doi.org/10.37569/DalatUniversity.9.2.531(2019)

Loại bài báo: Bài báo nghiên cứu gốc có bình duyệt

Bản quyền @ 2019 (Các) Tác giả.

Cấp phép: Bài báo này được cấp phép theo CC BY-NC-ND 4.0 


\title{
PHYTOSTEROLS, A TRITERPENOID, AND A LONG CHAIN ALCOHOL ISOLATED FROM THE LEAVES OF C amellia dalatensis Luong, Tran \& Hakoda
}

\author{
Nguyen Thi To Uyen ${ }^{a}$, Tran Thi Thanh Phuca, Luong Van Dung ${ }^{b}$, \\ Trinh Thi Diep ${ }^{\mathbf{a}^{*}}$ \\ ${ }^{a}$ The Faculty of Chemistry, Dalat University, Lamdong, Vietnam \\ ${ }^{b}$ The Faculty of Biology, Dalat University, Lamdong, Vietnam \\ *Corresponding author: Email: dieptt@dlu.edu.vn \\ Article history \\ Received: November $26^{\text {th }}, 2018$
}

Received in revised form: December $11^{\text {th }}, 2018 \mid$ Accepted: December $14^{\text {th }}, 2018$

\begin{abstract}
By various chromatographic methods, five compounds including spinasterol, stigmasterol, oleanolic acid, docosane, and 1-tricosanol were isolated from the ethanol extract of the leaves of Camellia dalatensis Luong, Tran, \& Hakoda. Their structures were elucidated by extensive spectroscopic methods including ID-NMR, 2D-NMR, ESI-MS, and IR. This is the first report of these compounds from this species.
\end{abstract}

Keywords: 1-tricosanol; Camellia dalatensis; Oleanolic acid; Spinasterol; Stigmasterol.

DOI: http://dx.doi.org/10.37569/DalatUniversity.9.2.531(2019)

Article type: (peer-reviewed) Full-length research article

Copyright (C) 2019 The author(s).

Licensing: This article is licensed under a CC BY-NC-ND 4.0 


\section{1. ĐặT VẤN ĐỀ}

Các loài thuộc chi Trà (Camellia) đã được con người sử dụng từ lâu đời làm đồ uống hàng ngày và để chữa bệnh. Trong số các loài Camellia, Trà xanh $(C$. sinensis $)$ được sử dụng rất phổ biến ở nước ta và trên thế giới. Trà xanh và các hợp chất polyphenol của nó đã được chứng minh có nhiều tác dụng đối với sức khỏe con người (Higdon \& Frei, 2003). Ngoài Trà xanh, hàng loạt các loài Camellia khác như $C$. japonica, $C$. oleifera, $C$. chrysantha, $C$. nitidssima ... cũng được báo cáo có chứa những thành phần hoạt chất quý giúp phòng chống ung thư (Dai \& ctg., 2016; Lambert \& Elias, 2010), hạ cholesterol máu (Maron \& ctg., 2003), giải độc gan, thận (Yokozawa, Chung, Young, Li, \& Oura, 1996). Các loài thuộc chi Camellia có thành phần hóa học khá phức tạp bao gồm nhiều thành phần như polyphenol, flavonoid, saponin, acid amin, phytosterol, các nguyên tố vi lượng (Balentine, 1997; Hara, Luo, Wickremashinghe, \& Yamanishi, 1995; \& Higdon \& Frei, 2003).

Trà Mi Đà Lạt (Camellia dalatensis Luong, Tran \& Hakoda) là một loài trà đặc hữu của Đà Lạt mới được nhóm nghiên cứu của Lương Văn Dũng, Nguyễn Văn Kết (Trường Đại học Đà Lạt), Trần Ninh (Trường Đại học Khoa học Tự nhiên, Đại học Quốc gia Hà Nội) và Hakoda (Nhật Bản) phát hiện và đặt tên khoa học vào năm 2012. Cho đển nay, loài trà này hầu như chưa được nghiên cứu về thành phần hóa học cũng như hoạt tính sinh học. Để góp phần làm rõ thành phần hóa học của loài trà này, chúng tôi đã nghiên cứu phân lập và xác định cấu trúc một số thành phần hóa học trong lá. Bài báo này trình bày kết quả phân lập và xác định cấu trúc của một số hợp chất trong dịch chiết $n$-hexan và dichloromethan.

\section{2. ĐỐI TƯợNG VÀ PHƯƠNG PHÁP NGHIÊN CÚU}

\section{1. Đối tượng nghiên cứu}

Lá Trà Mi Đà Lạt (Camellia dalatensis Luong, Tran \& Hakoda) được thu hái tại Phát Chi, Trạm Hành, Đà Lạt vào tháng 08 năm 2017. Mẫu nghiên cứu được thành viền của nhóm nghiên cứu là nhà thực vật học Lương Văn Dũng cung cấp, định danh tên khoa học và lưu tiêu bản (mã số DL.120401) tại Phòng Tiêu bản, Trường Đại học Đà Lạt.

\subsection{Phương pháp nghiên cứu}

\subsubsection{Phwơng pháp phân lập và xác định cấu trúc}

Phân lập các hợp chất bằng sắc ký cột và sắc ký lớp mỏng. Sắc ký lớp mỏng được thực hiện trên bản mỏng tráng sẵn silica gel $60 \mathrm{~F}_{254}$ (Merck). Thuốc thử hiện màu là dung dịch acid sulfuric $10 \%$ và đèn tử ngoại bước sóng 254 và $365 \mathrm{~nm}$. Sắc ký cột được thực hiện với chất hấp phụ là silica gel (cỡ hạt 40-63 $\mu \mathrm{m}$ và $63-200 \mu \mathrm{m}$, Merck). Xác định cấu trúc các hợp chất phân lập được dựa trên các thông số vật lý và các phương pháp phổ bao gồm: Điểm chảy, phổ hồng ngoại, phổ cộng hưởng từ hạt nhân, và phổ khối lượng. Điểm chảy được đo trên máy Yanaco MP-S3. Phổ IR được ghi trên 
máy Nicolet IS5 FT-IR. Phổ cộng hưởng từ hạt nhân ${ }^{1} \mathrm{H}-,{ }^{13} \mathrm{C}-\mathrm{NMR}$, DEPT, HSQC, và HMBC được ghi trên máy Bruker Avance-500 MHz, chuẩn nội TMS. Phổ khối ESI-MS được ghi trên máy Agilent 1100 LC-MSD Trap.

\subsubsection{Chiết xuất và phân lập}

Ba kilogam lá Trà Đà Lạt tươi đã được rửa sạch, cắt nhỏ chiết hồi lưu với 10 lít cồn $96 \%$ trong 2 giờ. Lọc lấy dịch chiết. Bã được chiết tiếp tương tự thêm ba lần, mỗi lần với 10 lít cồn $96 \%$. Các dịch chiết được gộp lại và thu hồi dung môi dưới áp suất giảm đến còn 1.6 lít dung dịch đậm đặc. Thêm một lít nước vào dung dịch đậm đặc và chiết phân đoạn lần lượt với các dung môi có độ phân cực tăng dần: n-hexan, dichloromethan, và ethyl acetat. Cất loại hết dung môi dưới áp suất giảm thu được các phân đoạn chiết tương ứng: n-hexan $(15.42 \mathrm{~g})$, dichloromethan $(9.72 \mathrm{~g})$, ethyl acetat $(13.58 \mathrm{~g})$, và nước $(177.05 \mathrm{~g})$.

Cắn chiết $n$-hexan $(15.42 \mathrm{~g})$ tiến hành chạy sắc ký cột với chất hấp phụ silica gel $(0.63-200 \mu \mathrm{m})$, rửa giải gradient với hệ dung môi $n$-hexan: Ethyl acetat thu được 11 phân đoạn được từ $\mathrm{H} 0$ đến $\mathrm{H} 10$. Phân đoạn $\mathrm{H} 3$ để kết tinh rồi kết tinh lại trong EtOAc thu được $13 \mathrm{mg}$ Hơp chất 1 . Phân đoạn $\mathrm{H} 5$ tiến hành chạy sắc ký cột silica gel (40 $63 \mu \mathrm{m}$, Merck) pha thường, rửa giải với hệ dung môi $\mathrm{CHCl}_{3}-$ EtOAc $(9: 1)$ thu được 12 phân đoạn từ $\mathrm{H} 5.1$ đến $\mathrm{H} 5.12$. Phân đoạn $\mathrm{H} 5.6$ được cô đến cắn và kết tinh lại trong EtOAc thu được $9 \mathrm{mg} H o ̛ ̣$ chất 2 . Phân đoạn $\mathrm{H} 5.10$ được cô đến cắn và kết tinh lại trong EtOAc thu được 8mg Hợp chất 3.

Cắn chiết dichloromethan $(9.72 \mathrm{~g})$ được phân tách bằng sắc ký cột thường với chất hấp phụ silica gel, rửa giải gradient với hệ dung môi $n$-hexan: Aceton theo độ phân cực tăng dần thu được sáu phân đoạn từ D1 đến D6. Phân đoạn D3 tiếp tục được phân tách bằng sắc ký cột silica gel, rửa giải với hệ dung môi EtOAc - MeOH $(10: 1)$ thu được 15 phân đoạn từ D3.1 - D3.15. Phân đoạn D3.1 kết tinh lại trong chloroform thu được 10mg Hợp chất 4. Phân đoạn D3.5 kết tinh lại trong chloroform thu được $7 \mathrm{mg}$ hợp chất 5 .

- Hợp chất 1: Tinh thể hình kim không màu, hiện màu vàng cam với TT $\mathrm{H}_{2} \mathrm{SO}_{4} 10 \%$. Nhiệt độ nóng chảy $168-169^{\circ} \mathrm{C}$. Phổ $\mathrm{IR}(\mathrm{KBr}): v_{\max }, \mathrm{cm}^{-1}$ : $3435(\mathrm{OH}) ; 2954,2869(\mathrm{CH}), 1659(\mathrm{C}=\mathrm{C})$. Phổ ESI-MS: $\mathrm{m} / z$ $411.1[\mathrm{M}-\mathrm{H}]^{-}$, $413.3[\mathrm{M}+\mathrm{H}]^{+}, \mathrm{M}=412\left(\mathrm{C}_{29} \mathrm{H}_{48} \mathrm{O}\right)$. Phổ ${ }^{1} \mathrm{H}-\mathrm{NMR}\left(500 \mathrm{MHz}, \mathrm{CDCl}_{3}\right) \delta$, ppm. Phổ ${ }^{13} \mathrm{C}-\mathrm{NMR}\left(125 \mathrm{MHz}, \mathrm{CDCl}_{3}\right) \delta$, ppm (Bảng 1).

- Hợp chất 2: Tinh thể hình kim không màu, hiện màu tím hồng với TT $\mathrm{H}_{2} \mathrm{SO}_{4} 10 \%$. Nhiệt độ nóng chảy $164-165^{\circ} \mathrm{C}$. Phổ IR $(\mathrm{KBr}): v_{\max }, \mathrm{cm}^{-1}$ : $3437(\mathrm{OH}) ; 2937,2860(\mathrm{CH})$. Phổ ESI-MS: $\mathrm{m} / z$, $413.0[\mathrm{M}+\mathrm{H}]^{+}, \mathrm{M}=412$ $\left(\mathrm{C}_{29} \mathrm{H}_{48} \mathrm{O}\right)$. Phổ ${ }^{1} \mathrm{H}-\mathrm{NMR}\left(500 \mathrm{MHz}, \mathrm{CDCl}_{3}\right) \delta$, ppm. Phổ ${ }^{13} \mathrm{C}-\mathrm{NMR}(125$ $\left.\mathrm{MHz}, \mathrm{CDCl}_{3}\right) \delta$, ppm (Bảng 1).

- Hợp chất 3: Tinh thể hình kim không màu, hiện màu hồng với $\mathrm{TT}^{\mathrm{H}} \mathrm{H}_{2} \mathrm{SO}_{4}$ $10 \%$. Nhiệt độ nóng chảy $308-310^{\circ} \mathrm{C}$. Phổ IR $(\mathrm{KBr}): v_{\max }, \mathrm{cm}^{-1}: 3432(\mathrm{OH})$; 
2926, $2854(\mathrm{CH}), 1691(\mathrm{C}=\mathrm{O})$. Phổ ESI-MS: $\mathrm{m} / z$ 457,1 $[\mathrm{M}+\mathrm{H}]^{+}, \mathrm{M}=456$ $\left(\mathrm{C}_{30} \mathrm{H}_{48} \mathrm{O}_{3}\right)$. Phổ ${ }^{1} \mathrm{H}-\mathrm{NMR}\left(500 \mathrm{MHz}, \mathrm{CDCl}_{3}\right) \delta$, ppm. Phổ ${ }^{13} \mathrm{C}-\mathrm{NMR}(125$ $\mathrm{MHz}, \mathrm{CDCl}_{3}$ ) $\delta$, ppm (Bảng 1).

- Hợp chất 4: Bột vô định hình màu trắng, nhiệt độ nóng chảy $44^{0} \mathrm{C}$. Phổ ${ }^{1} \mathrm{H}-$ $\operatorname{NMR}\left(500 \mathrm{MHz}, \mathrm{CDCl}_{3}\right) \delta$, ppm: $0.88(\mathrm{t}, J=7.0 \mathrm{~Hz}, 6 \mathrm{H}) ; 1.25(\mathrm{~m}, 40 \mathrm{H})$.

- Hợp chất 5: Bột vô định hình màu trắng, nhiệt độ nóng chảy $72^{\circ} \mathrm{C}$. Phổ ESI-MS: $m / z$ 341,1 $[\mathrm{M}+\mathrm{H}]^{+}, \mathrm{M}=340\left(\mathrm{C}_{23} \mathrm{H}_{48} \mathrm{O}\right)$. Phổ ${ }^{1} \mathrm{H}-\mathrm{NMR}(500 \mathrm{MHz}$, $\left.\mathrm{CDCl}_{3}\right) \delta$, ppm: 0.88 (t, $\left.J=7.0 \mathrm{~Hz}, 3 \mathrm{H}, \mathrm{H}-23\right) ; 1.25$ (m, 38H, H-4 - H-20); $1.31(\mathrm{~m}, 2 \mathrm{H}, \mathrm{H}-3) ; 1.56(\mathrm{~m}, 2 \mathrm{H}, \mathrm{H}-2) ; 3.64$ (t, $J=7.0 \mathrm{~Hz}, 2 \mathrm{H}, \mathrm{H}-1)$. Phồ ${ }^{13} \mathrm{C}-\mathrm{NMR}\left(125 \mathrm{MHz}, \mathrm{CDCl}_{3}\right) \delta$, ppm: $63.14(\mathrm{C}-1) ; 32.86(\mathrm{C}-2) ; 31.94(\mathrm{C}-$ 21); 29.37 - 29.71 (C-4 - C-20); 25.77 (C-3); 22.70 (C-22); 14.10 (C-23).

\section{KẾT QUẢ VÀ THẢO LUẬN}

Bảng 1. Số liệu phổ NMR của các Họ̣p chất 1, 2, và 3

\begin{tabular}{|c|c|c|c|c|c|c|}
\hline \multirow[b]{2}{*}{$\mathrm{C}$} & \multicolumn{2}{|c|}{ Hợp chất 1} & \multicolumn{2}{|l|}{ Hợp chất 2} & \multicolumn{2}{|c|}{ Hợp chất 3} \\
\hline & $\begin{array}{l}\delta_{C^{a, b}} \\
(\mathrm{ppm})\end{array}$ & $\begin{array}{l}\delta_{\mathrm{H}}^{\mathrm{c}, \mathrm{b}}(\mathrm{ppm}) \\
(J, \mathrm{~Hz})\end{array}$ & $\delta_{\mathrm{C}}^{\mathrm{a}, \mathrm{b}}(\mathrm{ppm})$ & $\begin{array}{l}\delta_{\mathrm{H}}^{\mathrm{c}, \mathrm{b}}(\mathrm{ppm}) \\
(J, \mathrm{~Hz})\end{array}$ & $\begin{array}{l}\delta_{C^{a, b}} \\
(\mathrm{ppm})\end{array}$ & $\begin{array}{l}\delta_{\mathrm{H}}^{\mathrm{c}, \mathrm{b}}(\mathrm{ppm}) \\
(J, \mathrm{~Hz})\end{array}$ \\
\hline 1 & 37.19 & $\begin{array}{l}1.81(\mathrm{~m}, 1 \mathrm{H}) \\
1.03(\mathrm{~m}, 1 \mathrm{H})\end{array}$ & 37.28 & & 38.44 & $1.61(\mathrm{~m}, 1 \mathrm{H})$ \\
\hline 2 & 31.53 & $\begin{array}{l}1.79(\mathrm{~m}, 1 \mathrm{H}) \\
1.38(\mathrm{~m}, 1 \mathrm{H})\end{array}$ & 31.62 & & 27.71 & $\begin{array}{l}1.08(\mathrm{~m}, 1 \mathrm{H}) \\
1.71(\mathrm{~m}, 1 \mathrm{H})\end{array}$ \\
\hline 3 & 71.09 & $3.59(\mathrm{~m}, 1 \mathrm{H})$ & 71.83 & $3.52(\mathrm{~m}, 1 \mathrm{H})$ & 79.06 & $\begin{array}{l}3.22(\mathrm{dd}, J=6.0 \\
11.0 \mathrm{~Hz}, 1 \mathrm{H})\end{array}$ \\
\hline 4 & 38.05 & $\begin{array}{l}1.70(\mathrm{~m}, 1 \mathrm{H}) \\
1.26(\mathrm{~m}, 1 \mathrm{H})\end{array}$ & 42.33 & & 38.78 & - \\
\hline 5 & 40.32 & $1.39(\mathrm{~m}, 1 \mathrm{H})$ & 140.78 & $5.35(\mathrm{~m}, 1 \mathrm{H})$ & 55.26 & $0.72(\mathrm{~m}, 1 \mathrm{H})$ \\
\hline 6 & 29.68 & $1.77(\mathrm{~m}, 2 \mathrm{H})$ & 121.71 & & 18.33 & $\begin{array}{l}1.54(\mathrm{~m}, 1 \mathrm{H}) \\
1.38(\mathrm{~m}, 1 \mathrm{H})\end{array}$ \\
\hline 7 & 117.49 & $5.15(\mathrm{br} \mathrm{s}, 1 \mathrm{H})$ & 31.92 & & 32.67 & $\begin{array}{l}1.30(\mathrm{~m}, 1 \mathrm{H}) \\
1.44(\mathrm{~m}, 1 \mathrm{H})\end{array}$ \\
\hline 8 & 139.59 & - & 31.92 & - & 39.31 & - \\
\hline 9 & 49.51 & $1.64(\mathrm{~m}, 1 \mathrm{H})$ & 50.19 & & 47.66 & $1.54(\mathrm{~m}, 1 \mathrm{H})$ \\
\hline 10 & 34.26 & - & 36.53 & - & 37.11 & - \\
\hline 11 & 21.59 & $\begin{array}{l}1.58(\mathrm{~m}, 1 \mathrm{H}) \\
1.46(\mathrm{~m}, 1 \mathrm{H})\end{array}$ & 21.09 & & 22.98 & $1,89(\mathrm{~m}, 1 \mathrm{H})$ \\
\hline 12 & 39.51 & $\begin{array}{l}1.99(\mathrm{~m}, 1 \mathrm{H}) \\
1.24(\mathrm{~m}, 1 \mathrm{H})\end{array}$ & 39.70 & & 122.67 & $\begin{array}{l}5,28(\mathrm{t}, J=3,5 \\
\mathrm{Hz}, 1 \mathrm{H})\end{array}$ \\
\hline 13 & 43.33 & - & 42.24 & - & 143.61 & - \\
\hline
\end{tabular}


Bảng 1. Số liệu phổ NMR của các Hợp chất 1, 2 , và 3 (tiếp theo)

\begin{tabular}{|c|c|c|c|c|c|c|}
\hline \multirow[b]{2}{*}{$\mathrm{C}$} & \multicolumn{2}{|c|}{ Hợp chất 1} & \multicolumn{2}{|l|}{ Hợp chất 2} & \multicolumn{2}{|c|}{ Hợp chất 3} \\
\hline & $\begin{array}{l}\delta_{C^{a}, \mathrm{~b}} \\
(\mathrm{ppm})\end{array}$ & $\begin{array}{l}\delta_{\mathrm{H}}^{\mathrm{c}, \mathrm{b}}(\mathrm{ppm}) \\
(J, \mathrm{~Hz})\end{array}$ & $\delta_{\mathrm{C}}^{\mathrm{a}, \mathrm{b}}(\mathrm{ppm})$ & $\begin{array}{l}\delta_{\mathrm{H}}^{\mathrm{c}, \mathrm{b}}(\mathrm{ppm}) \\
(J, \mathrm{~Hz})\end{array}$ & $\begin{array}{l}\delta_{\mathrm{C}}^{\mathrm{a}, \mathrm{b}} \\
(\mathrm{ppm})\end{array}$ & $\begin{array}{l}\delta_{\mathrm{H}}^{\mathrm{c}, \mathrm{b}}(\mathrm{ppm}) \\
(J, \mathrm{~Hz})\end{array}$ \\
\hline 14 & 55.17 & $1.81(\mathrm{~m}, 1 \mathrm{H})$ & 56.89 & & 41.66 & - \\
\hline 15 & 23.04 & $\begin{array}{l}1.50(\mathrm{~m}, 1 \mathrm{H}) \\
1.38(\mathrm{~m}, 1 \mathrm{H})\end{array}$ & 24.37 & & 27.21 & $1.59(\mathrm{~m}, 1 \mathrm{H})$ \\
\hline 16 & 28.51 & $\begin{array}{l}1.27(\mathrm{~m}, 1 \mathrm{H}) \\
1.72(\mathrm{~m}, 1 \mathrm{H})\end{array}$ & 28.91 & & 23.42 & $\begin{array}{l}0.87(\mathrm{~m}, 1 \mathrm{H}) \\
1.60(\mathrm{~m}, 1 \mathrm{H})\end{array}$ \\
\hline 17 & 55.97 & $1.24(\mathrm{~m}, 1 \mathrm{H})$ & 55.99 & & 46.53 & - \\
\hline 18 & 12.07 & $0.55(\mathrm{~s}, 3 \mathrm{H})$ & 12.06 & $1.01(\mathrm{~s}, 3 \mathrm{H})$ & 41.07 & $\begin{array}{l}2.82(\mathrm{dd}, J=4.0 \\
14 \mathrm{~Hz}, 1 \mathrm{H})\end{array}$ \\
\hline 19 & 13.05 & $0.80(\mathrm{~s}, 3 \mathrm{H})$ & 19.40 & $0.70(\mathrm{~s}, 3 \mathrm{H})$ & 45.92 & $1.16(\mathrm{~m}, 1 \mathrm{H})$ \\
\hline 20 & 40.81 & $2.04(\mathrm{~m}, 1 \mathrm{H})$ & 40.47 & & 30.69 & - \\
\hline 21 & 21.39 & $\begin{array}{l}1.03(\mathrm{~d}, J=6,5 \\
\mathrm{Hz}, 3 \mathrm{H})\end{array}$ & 21.21 & $\begin{array}{l}1.02(\mathrm{~d}, J=6.5 \\
\mathrm{Hz}, 3 \mathrm{H})\end{array}$ & 33.83 & $\begin{array}{l}1.22(\mathrm{~m}, 1 \mathrm{H}) \\
1.34(\mathrm{~m}, 1 \mathrm{H})\end{array}$ \\
\hline 22 & 138.17 & $\begin{array}{l}5.16(\mathrm{dd}, J=8.5 \\
15.0 \mathrm{~Hz}, 1 \mathrm{H})\end{array}$ & 138.31 & $\begin{array}{l}5.16(\mathrm{dd}, J=8.5 \\
15.0 \mathrm{~Hz}, 1 \mathrm{H})\end{array}$ & 32.46 & $\begin{array}{l}1.57(\mathrm{~m}, 1 \mathrm{H}) \\
1.76(\mathrm{~m}, 1 \mathrm{H})\end{array}$ \\
\hline 23 & 129.51 & $\begin{array}{l}5.03(\mathrm{dd}, J=8.5 \\
15.0 \mathrm{~Hz}, 1 \mathrm{H})\end{array}$ & 129.23 & $\begin{array}{l}5.02(\mathrm{dd}, J=8.5 \\
15.0 \mathrm{~Hz}, 1 \mathrm{H})\end{array}$ & 28.12 & $0.99(\mathrm{~s}, 3 \mathrm{H})$ \\
\hline 24 & 51.28 & $1.24(\mathrm{~m}, 1 \mathrm{H})$ & 51.25 & & 15.55 & $0.78(\mathrm{~s}, 3 \mathrm{H})$ \\
\hline 25 & 31.90 & $1.51(\mathrm{~m}, 1 \mathrm{H})$ & 31.88 & & 15.33 & $0.92(\mathrm{~s}, 3 \mathrm{H})$ \\
\hline 26 & 21.08 & $\begin{array}{l}0.85(\mathrm{~d}, J=6.5 \\
\mathrm{Hz}, 3 \mathrm{H})\end{array}$ & 18.99 & $\begin{array}{l}0.85(\mathrm{~d}, J=6.5 \\
\mathrm{Hz}, 3 \mathrm{H})\end{array}$ & 17.12 & $0.76(\mathrm{~s}, 3 \mathrm{H})$ \\
\hline 27 & 19.02 & $\begin{array}{l}0.83(\mathrm{~d}, J=6.5 \\
\mathrm{Hz}, 3 \mathrm{H})\end{array}$ & 21.07 & $\begin{array}{l}0.80(\mathrm{~d}, J=6.5 \\
\mathrm{Hz}, 3 \mathrm{H})\end{array}$ & 25.93 & $1.14(\mathrm{~s}, 3 \mathrm{H})$ \\
\hline 28 & 25.40 & $\begin{array}{l}1.41(\mathrm{~m}, 1 \mathrm{H}) \\
1.18(\mathrm{~m}, 1 \mathrm{H})\end{array}$ & 25.40 & & 182.59 & - \\
\hline 29 & 12.24 & $\begin{array}{l}0.81(\mathrm{t}, J=7.0 \\
\mathrm{Hz}, 3 \mathrm{H})\end{array}$ & 12.23 & $\begin{array}{l}0.81(\mathrm{t}, J=7.0 \\
\mathrm{Hz}, 3 \mathrm{H})\end{array}$ & 33.07 & $0.90(\mathrm{~s}, 3 \mathrm{H})$ \\
\hline 30 & & & & & 23.59 & $0.93(\mathrm{~s}, 3 \mathrm{H})$ \\
\hline
\end{tabular}

Ghi chú: ${ }^{\mathrm{a}} 125 \mathrm{MHz}$, ${ }^{\mathrm{b}}$ đo trong $\mathrm{CDCl}_{3},{ }^{\mathrm{c}} 500 \mathrm{MHz}$.

Hợp chất 1 thu được dưới dạng tinh thể hình kim màu trắng. Phổ IR của Hợp

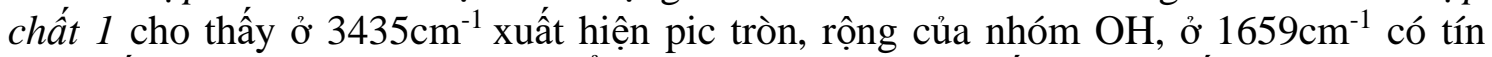
hiệu hấp thụ của nhóm $\mathrm{C}=\mathrm{C}$. Phổ ${ }^{1} \mathrm{H}-\mathrm{NMR}$ của Hợp chất 1 cho thấy tín hiệu cộng hưởng của 3 proton olefin ở $\delta_{\mathrm{H}} 5.16(\mathrm{dd}, 1 \mathrm{H}, J=8.5 ; 15.0 \mathrm{~Hz}), 5.15(\mathrm{br} \mathrm{s}, 1 \mathrm{H})$ và 5.03 $(\mathrm{dd}, 1 \mathrm{H}, J=8.5 ; 15.0 \mathrm{~Hz}$ ), một proton liên kết với $-\mathrm{C}-\mathrm{OH}$ ở $3.59(\mathrm{~m})$ và proton của 6 nhóm methyl ở $\delta 1.03(\mathrm{~d}, 3 \mathrm{H}, J=6.5 \mathrm{~Hz}), 0.85(\mathrm{~d}, 3 \mathrm{H}, J=6.5 \mathrm{~Hz}), 0.83(\mathrm{~d}, 3 \mathrm{H}, J=6.5$ $\mathrm{Hz}), 0.81(\mathrm{t}, 3 \mathrm{H}, J=7.0 \mathrm{~Hz}), 0.80(\mathrm{~s}, 3 \mathrm{H})$, và $0.55(\mathrm{~s}, 3 \mathrm{H})$. Tín hiệu proton methyl ở vùng trường cao tại $\delta_{\mathrm{H}} 0.55(\mathrm{~s}, 3 \mathrm{H})$ rất đặc trưng cho khung sterol (Panawan \& ctg., 


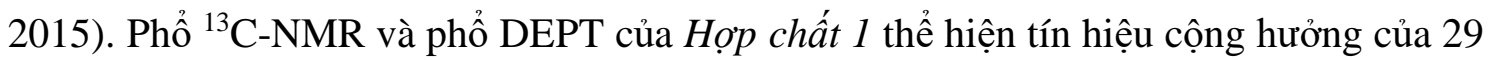
carbon, trong đó có 4 carbon olefin tại $\delta_{\mathrm{C}} 139.59 ; 138.17 ; 129.51$; và $117.49 \mathrm{ppm}, 1$ carbon liên kết với oxy tại $\delta_{\mathrm{C}} 71.09 \mathrm{ppm}, 7$ nhóm $\mathrm{CH}$ no, $2 \mathrm{C}, 9 \mathrm{CH}_{2}$, và 6 nhóm $\mathrm{CH}_{3}$. Đó là những tín hiệu cộng hưởng đặc trưng cho hợp chất sterol chứa một nhóm $\mathrm{OH}$ và 2 liên kết đôi. Từ các đặc trưng phổ nói trên, dự kiển Hợp chất 1 là một sterol có tên gọi là spinasterol. Các số liệu phổ NMR của Hợ chất 1 được so sánh với số liệu đã công bố cho spinasterol (Daiane $\&$ ctg., 2013) là hoàn toàn phù hợp. Các giá trị phổ ${ }^{1} \mathrm{H}$ - và ${ }^{13} \mathrm{C}-\mathrm{NMR}$ của các vị trí được gán chính xác nhờ vào các phổ hai chiều 2D-NMR. Phổ HSQC đã giúp xác định các giá trị độ dịch chuyển hoá học $\delta_{\mathrm{H}}$ từ các giá trị $\delta_{\mathrm{C}}$ của các carbon có mang proton trong cấu trúc và được thể hiện ở Bảng 1 . Các tương tác xa (HMBC) giữa $\mathrm{H}$ với $\mathrm{C}$ (Hình 2 ) khẳng định các giá trị độ dịch chuyển hóa học của các vị trí $\mathrm{C}$ và $\mathrm{H}$ như ở Bảng 1 là hoàn toàn phù hợp. Phổ ESI-MS của Hợp chất 1 với pic ion $[\mathrm{M}-\mathrm{H}]^{-}$tại $\mathrm{m} / z 411.1$ và pic ion $[\mathrm{M}+\mathrm{H}]^{+}$tại $\mathrm{m} / \mathrm{z} 413.3$ cho thấy khối lượng phân tử của Hợp chất 1 là $\mathrm{M}=412$, tương ứng với cổng thức phân tử $\mathrm{C}_{29} \mathrm{H}_{48} \mathrm{O}$ của spinasterol. Từ các bằng chứng phổ nói trên, Hơp chất 1 được xác định là $(3 \beta, 5 \alpha, 22 \mathrm{E})$-stigmasta7.22-dien-3-ol hay spinasterol. Hợp chất này đã được chứng minh có tác dụng giảm đau (Brusco \& ctg., 2017), hoạt tính độc tế bào đối với các dòng tế bào ung thư vú, ung thư tử cung (Meneses \& ctg., 2017; Sedky \& ctg., 2018).

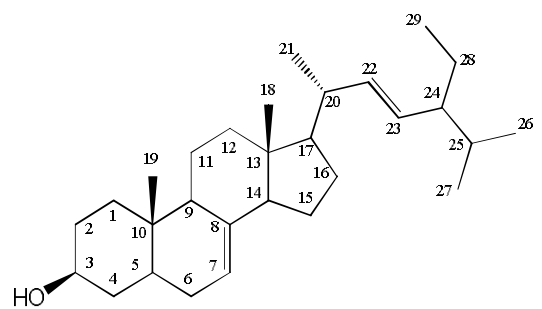

(a)

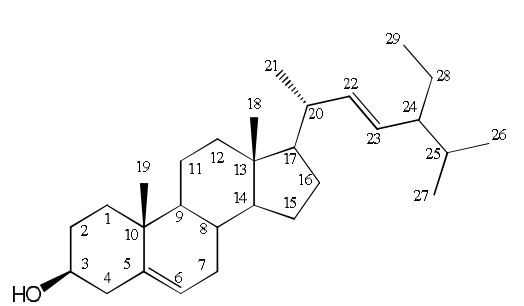

(b)

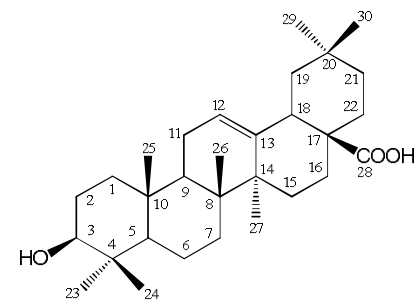

(c)<smiles>CCCCCCCCCCCCCCCCCCCCCCCCC</smiles>

(d)

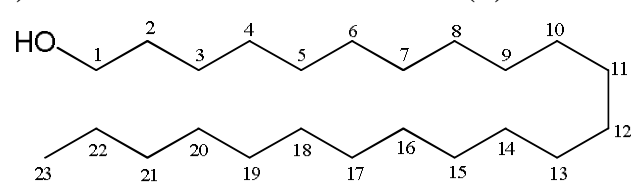

(e)

Hình 1. Cấu trúc hóa học của các họ̣p chất phân lập được Ghi chú: a) Hơp chất 1; b) Hợp chất 2; c) Hợp chất 3; d) Hợp chất 4; và e) Hợp chất 5 .

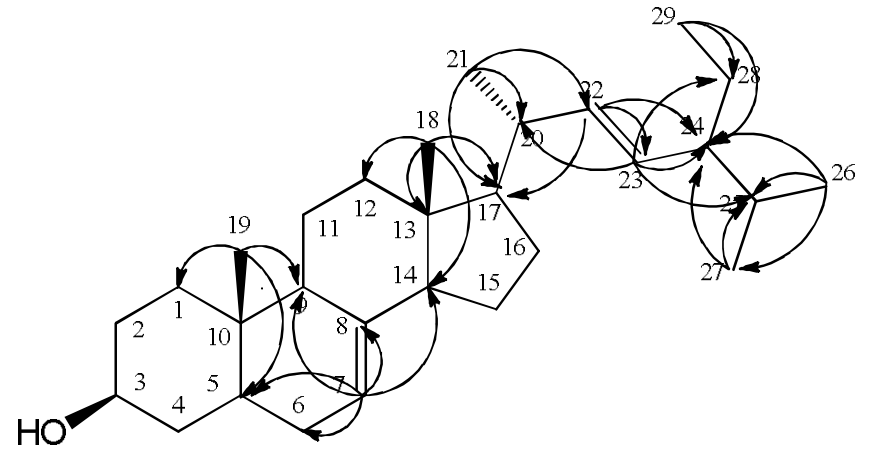

Hình 2. Các tương tác HMBC chính của Họp chất 1 
Hợp chất 2 có dạng tinh thể hình kim màu trắng, hiện màu tím hồng với TT $\mathrm{H}_{2} \mathrm{SO}_{4} 10 \%$, gợi ý hợp chất này cũng là một phytosterol. Các phổ NMR của Hợp chất 2 có nhiều tín hiệu giống với phổ của Hợp chất 1 . Phổ ${ }^{1} \mathrm{H}-\mathrm{NMR}$ cũng cho thấy tín hiệu cộng hưởng của 3 proton olefin, nhưng ngoài 2 proton olefin mạch hở dạng $\mathrm{HC}=\mathrm{CH}$ ở $\delta_{\mathrm{H}} 5.16(\mathrm{dd}, 1 \mathrm{H}, J=8.5 ; 15.0 \mathrm{~Hz})$ và $5.02(\mathrm{dd}, 1 \mathrm{H}, J=8.5 ; 15.0 \mathrm{~Hz})$, có một tín hiệu đặc trưng cho proton của liên kết đôi dạng $>\mathrm{C}=\mathrm{CH}$ ở $\mathrm{C} 5$ và $\mathrm{C} 6$ của khung stigmastan ở $\delta_{\mathrm{H}} 5.35(\mathrm{~m}, 1 \mathrm{H})$. Phổ ${ }^{1} \mathrm{H}-\mathrm{NMR}$ cũng cho thấy tín hiệu của một proton liên kết với -C$\mathrm{OH}$ ở $3.52(\mathrm{~m}, 1 \mathrm{H}, \mathrm{H}-3)$ và proton của 6 nhóm methyl ở $\delta 1.02(\mathrm{~d}, 3 \mathrm{H}, J=6.5 \mathrm{~Hz}), 1.01$ $(\mathrm{s}, 3 \mathrm{H}), 0.85(\mathrm{~d}, 3 \mathrm{H}, J=6.5 \mathrm{~Hz}), 0.81(\mathrm{t}, 3 \mathrm{H}, J=7.0 \mathrm{~Hz}), 0.80(\mathrm{~d}, 3 \mathrm{H}, J=6.5 \mathrm{~Hz})$, và $0.70(\mathrm{~s}, 3 \mathrm{H})$. Phổ ${ }^{13} \mathrm{C}-\mathrm{NMR}$ và phổ DEPT của Hơp chất 2 cũng thể hiện tín hiệu cộng hưởng của 29 carbon, trong đó có 4 carbon olefin tại $\delta_{\mathrm{C}} 140.78 ; 138.31 ; 129.31$; và $121.71 \mathrm{ppm}, 1$ carbon liên kết với $-\mathrm{OH}$ tại $\delta_{\mathrm{C}} 71.83 \mathrm{ppm}$ và 6 nhóm $\mathrm{CH}_{3}$. Từ các đặc trưng phổ nói trên, dự kiến Hợp chất 2 là một sterol có tên gọi là stigmasterol. Các số liệu phổ NMR của Hợp chất 2 (Bảng 1) được so sánh với số liệu đã công bố cho stigmasterol (Anjoo \& Ajay, 2011) là hoàn toàn phù hợp. Phổ ESI-MS của Hợp chất 2 cho thấy pic ion $[\mathrm{M}+\mathrm{H}]^{+}$tại $\mathrm{m} / z, 413.0$ phù hợp với công thức phân tử $\mathrm{C}_{29} \mathrm{H}_{48} \mathrm{O}, \mathrm{M}=$ 412. Từ các bằng chứng phổ nói trên, Hợp chất 2 được xác định là stigmasterol. Hợp chất sterol này phân bố khá rộng rãi trong thực vật, có nhiều trong dầu ăn và có nhiều tác dụng tốt cho sức khỏe con người như chống viêm (Antwi \& ctg., 2017), ức chế khối u (Kangsamaksin \& ctg., 2017).

Hợp chất 3 thu được dưới dạng tinh thể hình kim không màu. Phổ IR của Hợp chất 3 cho thấy ở $3432 \mathrm{~cm}^{-1}$ xuất hiện pic tròn, rộng của nhóm $\mathrm{OH}$, ở $1691 \mathrm{~cm}^{-1}$ có tín hiệu hấp thụ mạnh của nhóm $\mathrm{C}=\mathrm{O}$. Phổ ${ }^{1} \mathrm{H}-\mathrm{NMR}$ của Hợp chất 3 cho thấy sự có mặt của 7 nhóm methyl bậc ba $\left[\delta_{\mathrm{H}} 0.76(\mathrm{~s}, 3 \mathrm{H}) ; 0.78(\mathrm{~s}, 3 \mathrm{H}) ; 0.90(\mathrm{~s}, 3 \mathrm{H}) ; 0.92(\mathrm{~s}, 3 \mathrm{H}) ; 0.93\right.$ $(\mathrm{s}, 3 \mathrm{H}) ; 0.99(\mathrm{~s}, 3 \mathrm{H})$; và $1.14(\mathrm{~s}, 3 \mathrm{H})]$ thuộc khung triterpen olean. Một tín hiệu triplet ở $5.28(\mathrm{t}, J=3.5 \mathrm{~Hz}, 1 \mathrm{H})$ đặc trưng cho proton olefin $\mathrm{H}-12$ của khung triterpen 5 vòng. Phổ ${ }^{13} \mathrm{C}-\mathrm{NMR}$ và phổ DEPT của Hợp chất 3 cho thấy sự có mặt của 30 tín hiệu carbon, trong đó có 7 nhóm $\mathrm{CH}_{3}\left(\delta_{\mathrm{C}} 15.33 ; 15.55 ; 17.12 ; 23.59 ; 25.93 ; 28.12\right.$; và $\left.33.07 \mathrm{ppm}\right)$, 10 nhóm $\mathrm{CH}_{2}, 5$ nhóm $\mathrm{CH}$ và $8 \mathrm{C}$. Tín hiệu cộng hưởng tại $\delta_{\mathrm{C}} 182.59$ ppm cho thấy có một nhóm carboxylic $(\mathrm{COOH})$ trong cấu trúc. Các giá trị $\delta_{\mathrm{C}} 143.61$ và $122.67 \mathrm{ppm}$ khẳng định sự tồn tại của một liên kết đôi giữa $\mathrm{C}-12$ và $\mathrm{C}-13$. Một nhóm methin liên kết trực tiếp với nguyên tử oxy cộng hưởng ở $\delta_{\mathrm{C}} 79.06 \mathrm{ppm}$. Từ các đặc trưng phổ nói trên, dự kiến Hợp chất 3 là một triterpen có tên gọi là acid oleanolic. Các giá trị phổ ${ }^{1} \mathrm{H}$ - và ${ }^{13} \mathrm{C}-\mathrm{NMR}$ của các vị trí được gán chính xác nhờ vào các phổ hai chiều $\mathrm{HSQC}$ và $\mathrm{HMBC}$. Các tương tác $\mathrm{HMBC}$ quan trọng giữa $\mathrm{H}$ với $\mathrm{C}$ được thể hiện ở Hình 3 đã giúp khẳng định các giá trị độ dịch chuyển hóa học của các vị trí $\mathrm{C}$ và $\mathrm{H}$ như ở Bảng 1 . Phổ ESI-MS của Hơp chất 3 cho thấy pic ion $[\mathrm{M}+\mathrm{H}]^{+}$tại $\mathrm{m} / \mathrm{z} 457.1$ hoàn toàn phù hợp với công thức phân tử $\mathrm{C}_{30} \mathrm{H}_{48} \mathrm{O}_{3}$ của acid oleanolic, $\mathrm{M}=456$. Từ các bằng chứng phổ nói trên, Hơp chất 3 được xác định là acid oleanolic. Đây là hợp chất triterpen gặp trong nhiều loài cây thuốc và đang được chú ý về tiềm năng sử dụng trong điều trị các bệnh mãn tính (Ayeleso, Matumba, \& Mukwevho, 2017) và tác dụng ức chế khối u (Liu \& ctg., 2013). 


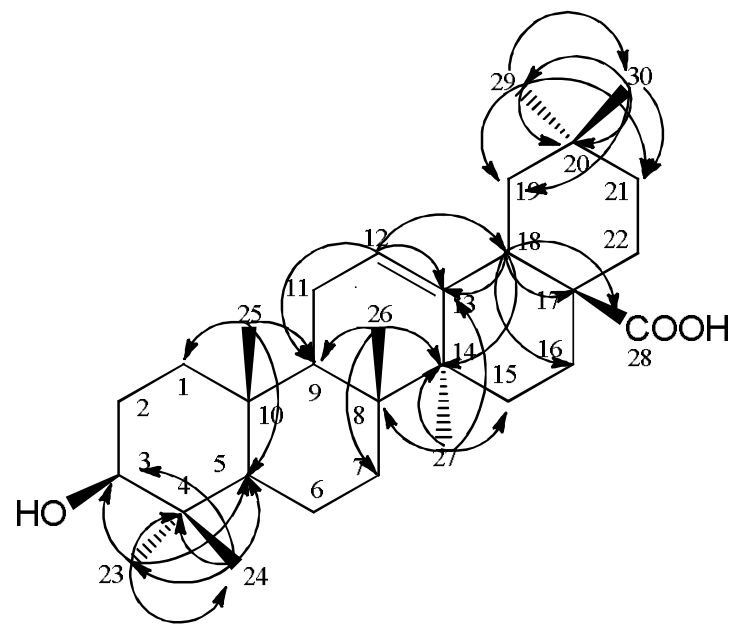

\section{Hình 3. Các tương tác HMBC chủ yếu của Hợp chất 3}

Hơp chất 4 là bột vô định hình màu trắng. Phổ ${ }^{1} \mathrm{H}-\mathrm{NMR}$ của $H o ̛ p$ chất 4 rất đơn giản, chỉ có một tín hiệu triplet của nhóm methyl tại $\delta_{\mathrm{H}} 0.88 \mathrm{ppm}(\mathrm{t}, J=7.0 \mathrm{~Hz}, 3 \mathrm{H})$ và cụm tín hiệu chồng chất của proton methylen tại $\delta_{\mathrm{H}} 1.25 \mathrm{ppm}(\mathrm{m}, 20 \mathrm{H})$. Đây là dạng phổ đặc trưng của một hydrocarbon no mạch thẳng có hai nhóm $\mathrm{CH}_{3}$ đầu mạch và 20 nhóm $\mathrm{CH}_{2}$. Như vậy, Hợp chất 4 có công thức phân tử là $\mathrm{C}_{22} \mathrm{H}_{46}$. Thêm vào đó, Hợp chất 4 nóng chảy ở $44^{\circ} \mathrm{C}$, hoàn toàn trùng khớp với nhiệt độ nóng chảy đã biết của docosan (Serghei, Kinza, Lydia, \& Heike, 2018). Điều đó góp phần khẳng định Hợp chất 4 chính là docosan.

Hợp chất 5 thu được dưới dạng bột vô định hình màu trắng, nhiệt độ nóng chảy $72^{0} \mathrm{C}$. Phổ ${ }^{1} \mathrm{H}-\mathrm{NMR}$ của Hợp chất 5 cho thấy tín hiệu proton của một nhóm methyl tại $\delta_{\mathrm{H}} 0.88 \mathrm{ppm}(\mathrm{t}, J=7.0 \mathrm{~Hz}, 3 \mathrm{H})$, một nhóm $\mathrm{CH}_{2} \mathrm{OH}$ tại $\delta_{\mathrm{H}} 3.64 \mathrm{ppm}(\mathrm{t}, J=7.0 \mathrm{~Hz}, 2 \mathrm{H})$ và cụm tín hiệu chồng chất của các proton methylen tại $\delta_{\mathrm{H}} 1.25 \mathrm{ppm}$. Phổ ${ }^{13} \mathrm{C}-\mathrm{NMR}$ của Hợp chất 5 một lần nữa khẳng định sự có mặt của một nhóm methyl tại $\delta_{\mathrm{C}} 14.10 \mathrm{ppm}$, nhóm $\mathrm{CH}_{2} \mathrm{OH}$ tại $\delta_{\mathrm{C}} 63.14 \mathrm{ppm}$ cùng với các nhóm $\mathrm{CH}_{2}$ trùng lấp nhau trong vùng $\delta_{\mathrm{C}}$ 29.37 - 29.71ppm. Như vậy, từ phổ NMR đã khẳng định được Hợp chất 5 có chứa một nhóm $\mathrm{CH}_{3}$, một nhóm $\mathrm{CH}_{2} \mathrm{OH}$. Phổ DEPT chỉ rõ tất cả các carbon còn lại đều là $\mathrm{CH}_{2}$. Như vậy Hơp chất 5 phải là một alcol no mạch thẳng. Phổ ESI-MS của Hợp chất 5 có pic ion phân tử $[\mathrm{M}+\mathrm{H}]^{+}$tại $m / z \quad 341.1$ tương ứng với công thức phân tử $\mathrm{C}_{23} \mathrm{H}_{48} \mathrm{O}$ của 1tricosanol, $\mathrm{M}=340$. Như vậy, từ các bằng chứng phổ nêu trên, Hơp chất 5 được xác định là 1-tricosanol.

\section{KẾT LUẬN}

Từ dịch chiết n-hexan và dichloromethan của lá Trà Mi Đà Lạt Camellia dalatensis Luong, Tran \& Hakoda, bằng các phương pháp sắc ký kết hợp với các phương pháp phồ hiện đại (IR, ${ }^{1} \mathrm{H}-\mathrm{NMR},{ }^{13} \mathrm{C}-\mathrm{NMR}$, DEPT, HSQC, HMBC, ESI-MS) đã phân lập và nhận dạng được cấu trúc năm hợp chất là spinasterol, stigmasterol, acid oleanolic, docosan, và 1-tricosanol. Đây là lần đầu tiên các hợp chất này được phân lập từ loài $C$. dalatensis. 


\section{TÀI LIỆU THAM KHẢO}

Anjoo, K., \& Ajay, K. S. (2011). Isolation of stigmasterol and $\beta$-sitosterol from petroleum ether extract of aerial parts of Ageratum conyzoides (Asteraceae). International Journal of Pharmacy and Pharmaceutical Sciences, 3, 94-96.

Antwi, A. O., Obiri, D. D., Osafo, N., Forkuo, A. D., \& Essel, L. B. (2017). Stigmasterol inhibits lipopolysaccharide-induced innate immune responses in murine models. International Immunopharmacology, 53, 105-113.

Ayeleso, T. B., Matumba, M. G., \& Mukwevho, E. (2017). Oleanolic acid and its derivatives: Biological activities and therapeutic potential in chronic diseases. Molecules, 22(11), 1-16.

Balentine, D. A. (1997). Introduction: Tea and health. Critical Reviews in Food Science and Nutrition, 8, 691-669.

Brusco, I, Camponogara, C., Carvalho, F. B., Schetinger, M. R. C., Oliveira, M. S., Trevisan, G., Ferreira, J., \& Oliveira, S. M. (2017). $\alpha$-Spinasterol: A COX inhibitor and a transient receptor potential vanilloid 1 antagonist presents an antinociceptive effect in clinically relevant models of pain in mice. British Journal of Pharmacology, 174(23), 4247-4262.

Consolacion, Y. R., Richard, F. G. , Mitzell, A., Vernadette, T., \& Chien, C. S. (2014). Triterpenes and sterols from Samanea saman. Research Journal of Pharmaceutical, Biological and Chemical Sciences, 5(4), 1501-1507.

Dai, L., Li, J. L., Liang, X. Q., Li, L., Feng, Y., Liu, H. Z., \& Zhang, L. T. (2016). Flowers of Camellia nitidissima cause growth inhibition, cell-cycle dysregulation and apoptosis in a human esophageal squamous cell carcinoma cell line. Molecular Medicine Reports, 14(2), 1117-1122.

Daiane, M., Lillian, L. C., Daniela, F. R., Kahlil, S. S., Pedro, E. A., Silva, A. B., \& Cecilia, V. N. (2013). Triterpenes and the antimycobacterial activity of Duroia macrophylla huber (Rubiaceae). BioMed Research International, 2013, 1-8.

Hara, Y., Luo, S. J., Wickremashinghe, R. L., \& Yamanishi, T. V. (1995). Chemical composition of tea. Food Reviews International, 11(3), 435-456.

Higdon, J. V., \& Frei, B. (2003). Tea catechins and polyphenols: Health effects, metabolism, and antioxidant functions. Critical Reviews in Food Science and Nutrition, 43(1), 89-143.

Kangsamaksin, T., Chaithongyot, S., Wootthichairangsan, C., Hanchaina, R., Tangshewinsirikul, C., \& Svasti, J. (2017). Lupeol and stigmasterol suppress tumor angiogenesis and inhibit cholangiocarcinoma growth in mice via downregulation of tumor necrosis factor- $\alpha$. PLoS One, 12(12), 1-16.

Lambert, J. D., \& Elias, R. J. (2010). The antioxidant and pro-oxidant activities of green tea polyphenols: A role in cancer prevention. Archives of Biochemistry and Biophysics, 501, 65-72. 
Liu, Q., Liu, H., Zhang, L., Guo, T., Wang, P., Geng, M., \& Li, Y. (2013). Synthesis and antitumor activities of naturally occurring oleanolic acid triterpenoid saponins and their derivatives. European Journal of Medicinal Chemistry, 64, 1-15.

Maron, D. J., Lu, G. P., Cai, N. S., Wu, Z. G., Li, Y. H., Zhu, J. Q., Jin, X. J., Wouters, B. C., Zhao, J., \& Chen, H. (2003). Cholesterol-lowering effect of a theaflavinenriched green tea extract: A randomized controlled trial. The Archives of Internal Medicine, 163(12), 1448-1453.

Meneses, S. S., Navarro, N. M., Ruiz, B. E., Del, T. S., Jiménez, E. M., \& Robles, Z. R. E. (2017). Antiproliferative activity of spinasterol isolated of Stegnosperma halimifolium. Saudi Pharmaceutical Journal, 25(8), 1137-1143.

Panawan, S., Watcharapong, C., Sugunya, M., Suwaporn, L., Somsuda, T., \& Vijittra, L. (2015). Structures of phytosterols and triterpenoids with potential anti-cancer activity in bran of black non-glutinous rice. Nutrients, 7, 1672-1687.

Sedky, N. K., El-Gammal, Z. H., Wahba, A. E., Mosad, E., Waly, Z. Y., El-Fallal, A. A., Arafa, R. K., \& El-Badri, N. (2018). The molecular basis of cytotoxicity of $\alpha$-spinasterol from Ganoderma resinaceum: Induction of apoptosis and overexpression of p53 in breast and ovarian cancer cell lines. Journal of Cellular Biochemistry, 119(5), 3892-3902.

Serghei, A., Kinza, S., Lydia, W., \& Heike, P. K. (2018). Effect of alkane chain length on crystallization in emulsions during supercooling in quiescent systems and under mechanical stress. Processes, 6(1), 1-6.

Yokozawa, T., Chung, H., Young, H., Li, Q., \& Oura, H. (1996). Effectiveness of green tea tannin on rats with chronic renal failure. Bioscience, Biotechnology, and Biochemistry, 60, 1000-1005. 